\title{
COVID-19 y el sistema renina, angiotensina, aldosterona. Una relación compleja
}

\author{
COVID-19 and the renin, angiotensin, aldosterone system. A complex relationship
}

Luis Alcocer-Díaz-Barreiro 1,2,4*, Jorge Cossio-Aranda,3, Juan Verdejo-Paris',3, Manuel Odin-de-los-Ríos², Héctor Galván-Oseguera ${ }^{4,5}$, Humberto Álvarez-López ${ }^{6}$ y Marco A. Alcocer-Gamba ${ }^{1}$

${ }^{1}$ Instituto Mexicano de Salud Cardiovascular, Interamerican Society of Hypertension; ${ }^{2}$ Sociedad Mexicana de Cardiología; ${ }^{3}$ Instituto Nacional de Cardiología "Ignacio Chávez"; ${ }^{4}$ Grupo de Expertos en Hipertensión Arterial. GREHTA México; ${ }^{5}$ Hospital de Cardiología, Centro Médico Nacional Siglo XXI, Instituto Mexicano del Seguro Social. Ciudad de México; ${ }^{6}$ Centro Médico Puerta de Hierro Andares en Zapopan, Guadalajara, Jalisco

\begin{abstract}
Resumen
La pandemia por COVID-19 ha tenido muy importantes repercusiones negativas, sanitarias, psicológicas, sociales y económicas para las personas, las familias, las comunidades, los países y para las para la humanidad en general. La interrelación con la edad y la presencia de enfermedades crónicas no trasmisibles (hipertensión, diabetes, obesidad, tabaquismo) parece ir mas lejos que lo que explicaría la prevalencia y distribución de ambas. Los medicamentos que actúan sobre el sistema renina-angiotensina-aldosterona, son pilares básicos en el manejo de estas enfermedades. Se sabe de tiempo atrás que estos fármacos aumentan en forma significativa la expresión en el tejido pulmonar de receptores para la enzima de conversión de angiotensina de tipo 2. Este hecho junto con el conocimiento de que la vía de entrada del virus a la célula es precisamente el receptor de ECA-2, inició una hipótesis, basada en evidencia de muy baja calidad, que rápidamente se generalizó en los medios de comunicación, de que el empleo de estos medicamentos podría ser negativo y que deberían suspenderse. La respuesta de prácticamente todas las sociedades científicas fue casi inmediata, con la indicación precisa de que no debería suspenderse el tratamiento con estos fármacos, puesto que la evidencia de su utilidad está basada en una evidencia muy sólida y de gran calidad. Casi simultáneamente también apareció la hipótesis, también basada en evidencia muy preliminar, de que estos medicamentos no solo resultan dañinos sino que son benéficos, tampoco se aceptan todavía como agentes para la prevención o tratamiento de esta enfermedad o sus complicaciones. La presente revisión relata los conocimientos actuales sobre la relación entre COVID-19 y SRAA.
\end{abstract}

Palabras clave: COVID-19. Sistema renina angiotensina aldosterona. Enzima de conversión de angiotensina-2. Coronavirus SARS- CoV-2 2019

\begin{abstract}
The COVID-19 pandemic has had major negative health, psychological, social and economic repercussions for individuals, families, communities, countries and for humanity in general. The interrelation with age and the presence of chronic non-communicable diseases (hypertension, diabetes, obesity, smoking) seems to go further than what would be explained by the prevalence and distribution of both. The drugs that act on the renin-angiotensin-aldosterone system are in many cases the backbone for the management of these diseases, it has been known for a long time that these drugs significantly increa-
\end{abstract}

${ }^{*}$ Correspondencia:

Luis Alcocer-Díaz-Barreiro

E-mail: alcocerdb @gmail.com
Disponible en internet: 26-05-2020 Arch Cardiol Mex. 2020;90(Supl):19-25 www.archivoscardiologia.com 1405-9940/@ 2020 Instituto Nacional de Cardiología Ignacio Chávez. Publicado por Permanyer. Este es un artículo open access bajo la licencia CC BY-NC-ND (http://creativecommons.org/licenses/by-nc-nd/4.0/). 
se the expression of receptors for angiotensin conversion enzyme type 2 in the lung tissue. This fact, together with the knowledge that the route of entry of the virus into the cell is precisely the ACE-2 receptor, initiated a hypothesis, based on very low-quality evidence, which quickly became generalized in the media, that the use of these drugs could be negative and that they should be interrupted immediately. The response of practically all Scientific Societies was almost immediate, with the precise indication that treatment with these drugs should not be discontinued, since the evidence of their usefulness is based on very solid and high-quality evidence. Simultaneously, a different hypothesis also appeared, also based on very preliminary evidence, that these drugs are not only harmful but also beneficial, however these medicaments are not yet accepted as agents for the prevention or treatment of this disease or its complications. This review reports current knowledge on the relationship between COVID-19 and SRAA.

Key words: COVID-19. Renin-angiotensin-aldosterone system. Angiotensin conversion enzyme type 2. Coronavirus SARS- COV-2 2019.

\section{Introducción}

La pandemia global de la enfermedad por infección del coronavirus SARS- CoV-2 2019 (COVID-19), ha tenido implicaciones negativas de magnitud sin precedentes para la salud biológica, psicológica y social de las personas. El fenómeno ha impactado gravemente en la economía de las familias y de los países, esto ha profundizado de manera grave las diferencias políticas prexistentes; y a la vez, como sucede con todo fenómeno que afecte al hombre, ha influido favorablemente en el progreso de la ciencia. La secuenciación genética del virus, la búsqueda de una vacuna y de un tratamiento se han hecho a una velocidad nunca vista.

Un aspecto interesante de las discusiones científicas es que ha resurgido el interés por el conocimiento de los mecanismos de acción viral y ha tomado importancia reciente, el recordar que la penetración del virus al interior de la célula, especialmente del endotelio pulmonar, pero también renal, intestinal y de los vasos sanguíneos, se hace por el camino del receptor de la enzima de conversión de angiotensina tipo 2 (ECA-2) ${ }^{1}$ y esto ha dado lugar a discusiones sobre la pertinencia del empleo de los medicamentos que actúan sobre el sistema renina angiotensina aldosterona (SRAA), en condiciones de la pandemia actual.

Es motivo de esta revisión, el destacar la bases científicas de estas discusiones en ocasiones controversiales y el relatar y analizar las recomendaciones emitidas por diferentes grupos y sociedades cardiológicas sobre el tema.

\section{Material y métodos}

Se realizó revisión de la literatura en PubMed en el periodo comprendido entre el 1 de diciembre de 2019 y el 14 de abril de 2020, con la palabra COVID-19, combinada alternativamente con ACE-2, ACE-Inhibitors, ARB y RAAS.

Se utilizaron los buscadores Google y Yahoo! para recabar información referente a los términos COVID-19 and RASS y en Scholar Google con los términos COVID-19 and antihypertensive treatment.

\section{Resultados}

En el periodo señalado se encontraron 120 publicaciones indexadas, con la siguiente distribución:con el término COVID-19 and ACE-2 (74 resultados), con COVID-19 and ACE-INHIBITORS (35 resultados), con COVID-19 and ARB (6 resultados), con COVID-19 and RAAS (4 resultados). La búsqueda de los términos COVID and ECA-2 en Google arroja 184 millones de menciones, En Yahoo! 228 millones. En Scholar Google 1,890 menciones. La revisión en Google se ajusta más a la presencia de ambos términos en forma simultanea, mientras que en Yahoo! se reportan además resultados de cada uno de los términos por separado. En Google Scholar se recogen los resultados de publicaciones de alto impacto, pero también en lo que podríamos llamar la literatura gris: resúmenes, libros y artículos de fuentes de calidad como editoriales académicas, sociedades profesionales, publicacionesd en linea preliminares o bien universidades.

La más extensa y completa revisión actual sobre el tema es la realizada por Sparks MA y colaboradores en el Blog NephJC22, que registra solamente 11 estudios sobre la severidad de la COVID-19 y su mortalidad en pacientes hipertensos, 9 de ellos en Wuhan, China, 1 en Italia y otro en Washington, USA. Reporta también 4 estudios sobre desenlaces clínicos con el uso de ARB y ACE en pacientes con COVID-19 en bases de 
datos de ciudades chinas. Todos estos estudios comprenden pocos casos y son retrospectivos. Registra también la posición recomendada respecto a la continuación o suspensión del tratamiento en pacientes tomando medicamentos que actúan en el SRAASRA de 13 sociedades científicas de Cardiología, Hipertensión, Nefrología, Diabetes, Insuficiencia Cardiaca, Medicina General y Pediatría, todas ellas publicadas en marzo de 2020.

Otro cambio importante en la vida científica, provocado por la pandemia, es el hecho de que las revistas médicas más prestigiadas, han acelerado muy significativamente su tiempo de aceptación de trabajos, han hecho casi universal la publicación inmediata en línea y el acceso sin costo a los artículos.

\section{Discusión}

La interrelación entre la intervención terapéutica sobre el SRAA y la COVID-19 se ha prestado a una intensa discusión reciente, por lo que pensamos pertinente recordar algunos conceptos básicos.

\section{Relación entre ECA-2 y Virus SARS-CoV2 (COVID-19)}

Debido a que se ha publicado que el virus SARSCoV2 requiere formar un complejo con el receptor ECA-2 para penetrar al interior de la célula endotelial principalmente a nivel pulmonar, apareció enseguida la preocupación relacionada a el uso de inhibidores de la enzima convertidora de angiotensina (IECA) o bloqueadores del receptor de angiotensina (BRAT) ya que se conoce que, por lo menos en animales que ambos fármacos incrementan, el número de receptores ECA2. Al existir un mayor número de receptores ECA-2, en teoría se facilitaría primeramente la infección celular por el virus y lo que es, aún peor, que la gravedad de la infección pudiera ser mayor en los pacientes tratados con estos fármacos.

Como se sabe, actualmente la hipertensión arterial es una de las principales co-morbilidades en los pacientes graves con COVID-19 y millones de pacientes están siendo tratados con IECA o BRAT, lo que provocó que algunos autores de gran prestigio ${ }^{3}$ externaron sus opiniones segidos por publicaciones no médicas que mostraron su preocupación al respecto, tal y como sucedión con el influyente Daily Mail de Inglaterra del 13 de marzo de $2020^{4}$, en el que con un lenguaje impreciso se sugerió indicar la necesaria interrupción de estos medicamentos.
La noticia se hizo rápidamente viral y en las redes sociales y medios masivos de comunicación como la radio o televisión, se recomendó en forma anticipada a los pacientes interrumpir el uso de IECA y BRAT (aún sin consultar al médico tratante). Existieron opiniones diversas y bastante heterogéneas, desde las que sugerían suspender el tratamiento a todos los pacientes no infectados tratados con estos fármacos independientemente de su indicación terapéutica (hipertensión arterial, nefropatía, insuficiencia cardiaca, etc.), hasta las que sugerían que solo deberían suspenderlo los pacientes infectados o por lo menos aquellos infectados que requierieran cuidados intensivos. Esta afirmación, como explicaremos más ampliamente, es en realidad meramente especulativa, pues no existe a la fecha evidencia clínica alguna, sobre la existencia de riesgos de adquirir o potencializar la infección, ni sobre la conducta de retirar estos medicamentos en quienes están controlados con ellos, por el contrario esta conducta podría resultar en mayor morbimortalidad cardiovascular debido al descontrol de la hipertensión arterial o enfermedad cardiovascular subyacente. De especial preocupación son los pacientes con insuficiencia cardiaca que se encuentran controlados con este tipo de fármacos y en los cuales constituye una terapia irreemplazable.

Resulta pués pertinente revisar los conocimientos actuales sobre el sistema renina-angiotensina-aldosterona (SRAA), las características del virus y la información sobre el efecto del bloqueo del SRAASRA en especial con inhibidores de la enzima de conversión de la angiotensina (IECA) y los bloqueadores de los receptores de tipo 1 de angiotensina II (BRAT).

\section{Sistema renina-angiotensina-aldosterona (SRAA)}

El sistema renina-angiotensina-aldosterona (SRAA) es un complejo y multifactorial sistema de péptidos y enzimas, que participan en múltiples acciones muy protagónicas en el organismo.

Recordemos en que consiste este complejo sistema, pilar fundamental en el sostenimiento de sistemas homeostáticos tan importantes como son el ajuste de la relación entre el continente del aparato circulatorio (vasos sanguíneos) y su contenido (volumen circulante), vitales para el funcionamiento del aparato circulatorio (gasto cardiaco y presión arterial) y para el equilibrio electrolítico en los tres compartimentos del cuerpo (intravascular, intersticial e intracelular).

El sustrato fundamental del SRAA lo constituye un péptido de catorce aminoácidos presente 
primordialmente en el hígado (SRAA circulante), aunque también puede encontrarse en prácticamente cualquier célula (SRAA local), sobre el cual actúa una enzima proteolítica, conocida como renina que es principalmente producida en la mácula densa renal, como resultado de múltiples estímulos en especial por la "percepción" de un flujo renal disminuido, un tono simpático aumentado y/o un desequilibrio electrolítico.

La renina acorta al tetradecapéptido angiotensinógeno en un decapéptido conocido como angiotensina I (At-I) que tiene poca actividad biológica. La enzima de conversión de la angiotensina (ECA), presente en la membrana de las células endoteliales (existe también una versión soluble circulante y probablemente una intracelular), convierte a la At-I, en un octapéptido conocido como angiotensina II (At-II) que ejerce múltiples acciones biológicas al unirse a su receptor conocido como RAt-1 y que produce respuestas fundamentalmente vasoconstrictoras, protrombóticas, proinflamatorias, de retención de agua y sodio que en condiciones fisiológicas participan eficientando el gasto cardiaco, redistribuyéndolo hacia las zonas que en determinado momento requieren mayor 0 menor consumo energético y que en condiciones de hipovolemia reduce el tamaño del sistema arterial, para mantener una presión y un flujo adecuados, y que retiene agua y sodio para restarurar el volumen. En caso de ruptura del sistema circulatorio, la angiotensina II intenta cerrarlo inicialmente con vasoconstricción y trombosis del sitio y posteriormente repararándola con sus mecanismos inflamatorios y proliferativos. Este sistema vital para el ser humano (que en un criterio evolucionista permitió a las especies iniciales abandonar el mar), cuando se sobre-expresa por un estimulo equivocado o por una falla intrínseca, se convierte en uno de los principales mecanismos fisiopatológicos para enfermedades tan importantes como la hipertensión arterial, la aterosclerosis, la diabetes y sus temibles consecuencias: la cardiopatía isquémica, la insuficiencia cardiaca y los daños renal, cerebral y hepático.

Una acción importante de la angiotensina II sobre el receptor RAt-1, es la estimulación de la sintasa de aldosterona, con lo que se convierte en uno de los más importantes secretagógos de la aldosterona, sustancia con profundos efectos sobre el equilibrio electrolítico y con efectos antiproliferativos muy potentes (especialmente antifibrosis).

En resumen el SRA es fundamental para la vida, pero es además uno de los principales mecanismos que explican, sostienen y complican a las principales enfermedades crónicas no trasmisibles que constituyen el principal problema de salud para la especie humana.

Como sucede con todo fenómeno o sistema biológico, el SRAA convive e interactúa con otros sistemas antagónicos. El primer sistema antagonico a SRAA reconocido historicamente, es el sistema de la bradiquinina, substancia que ejerce precisamente un efecto contrario al de la At-II: es un potente vasodilatador, estimulador de la producción de óxido nítrico (NO), que ejerce acciones antinflamatorias, antiproliferativas y antitrombóticas. Sucede que la ECA tambien tiene como acción enzimática el degradar a la bradiquinina, de tal forma que el aumento de la actividad de la ECA resulta por un lado en una mayor disponibilidad de angiotensina II, pero a su vez en menores cantidades de bradiquinina.

Otro sistema antagónico a la angiotensina II, es la existencia de receptores RAt-2, que si bién no están en todos los tejidos ni son constitutivos, (esto es, que solo se expresan por ejemplo en condiciones de estrés), su estimulación por variantes de la angiotensina II $\left(\beta-\mathrm{Tyr}^{4}\right.$ Angiol I y $\beta$-lle 5 -Angll) y por LP2-3 o C21, produce efectos contrarios a los de la estimulación del RAt-1.

El proceso de degradación de la angiotensina por enzimas proteolíticas es muy variado: la acción de la aminopeptidasa A, convierte a la angiotensina en un heptapéptido que pierde el primero y los dos últimos componentes de la cadena peptídica, por lo que se le clasifica como angiotensina 2-8 y se conoce como angiotensina III la que a su vez por acción de la aminopeptidasa $N$ se convierte en angiotensina 3-8, conocida como angiotensina IV, estos dos últimos subproductos son activos, la angiotensina III con acción similar a la At-I sobre los receptores RAt-1 y RAt-2 mientras que la Ang-IV actúa en una aminopeptidasa regulada por insulina (IRAP por sus siglas en inglés).

Más recientemente se describió la existente de una variante de la ECA, que es la ECA2 que es muy similar a la ECA pues conserva un $60 \%$ de homología con ella, su acción es convertir a la Ang-II en angiotensina 1-7 (Ang 1-7) que actúa sobre otro receptor de la membrana conocido como MAS, que se expresa en varios tejidos, entre los que sobresalen los neumocitos tipo 2 (productor del surfactante pulmonar), este péptido tiene una acción vasodilatadora y diurética de agua y sodio, con lo que resulta en un efeco moderadamente reductor de la presión arterial y al parecer más importante por estimular la producción de óxido nítrico por la célula endotelial, que resulta particularmente protector en especial del tejido alveolar.

Es relevante para esta revisión el hacer hincapíe en que la expresión de ECA2 se incrementa sustancialmente en pacientes con diabetes tipo 1 o tipo 2 y con el uso de 
IECA y BRAT y con los bloqueadores de mineralo corticoides, (al menos con espironolactona) ${ }^{5}$ además debemos recordar que ECA2 es una peptidasa unida a la membrana epitelial, que tiene en una posición extracelular en la mayoría de su dominio peptídico $\mathrm{N}$ terminal, que es su sitio catalítico y que a su vez es el que se une con los virus del grupo SARS al que pertenece el SARS-coronavirus- $2^{6}$

\section{Características relevantes del Virus SARS-cov-2 para su relación con SRA}

El virus SARS-CoV-2 causante de la actual pandemia de la COVID-19, que se inició a finales de 2019 en la ciudad china de Wuhan, es un coronavirus de la familia de los beta coronavirus, similar a otros virus que infectan a los murciélagos, y de la que tambien forman parte el virus del SARS (síndrome respiratorio severo agudo) y el agente causal del MERS (síndrome respiratorio de Medio Oriente, así como 4 coronavirus asociados a la gripe común. La secuencia genética de este virus fue rapidamente estudiada y hecha pública, en los primeros dias de enero ${ }^{7}$, al inicio de la pandemia.

SARS-CoV-2 es un virus de una cadena simple de RNA de polaridad positiva, es como los de su familia muy grande, pues mide 80-120 nm. Su genoma está integrado por aproximadamente 29,891 bases que codifican para 9860 aminoácidos. Está formado por tres proteínas estructurales: espiga (S), envoltura $(E)$ y membrana. La proteína estructural $S$ es una proteina altamente glicosilada y en asociación con otros homotrímeros estructura a las espigas tan características del virus y es la responsable de permitir el ingreso del COV-19 a las células alveolares de pulmón ya que determina el tropismo por el receptor ECA2.

En otros virus son estas espigas las que son el blanco de los antivirales, que en elcaso del agente causa de la COVID-2 han sido tres los probados sin que se demestre acción útil. Son además estas proteinas el principal objetivo para ser atacado con una vacuna que se busca intensamente.

Cada monómero de la proteína $\mathrm{S}$ se organiza en dos dominios: el S1 y el S2, en el S1 se encuentra el sitio de unión al receptor ECA-2 que se expresa en la subpoblación de células alveolares de tipo 2 que son las encargadas de producir el surfactante pulmonar, éste también es el sitio de entrada de otros SARS-CoV, mientras que los virus MERS-CoV usan la dipeptidil-peptidasa (DPP)-4 como receptor de superficie. Es probable además que el SARS-CoV-2 utilice otros receptores para infectar macrófagos y células $\mathrm{T}^{8}$.
Los virus para subsistir requieren crear incontables copias de si mismos y para esto se valen de la maquinaria intracelular, por lo que requieren infectar a un huésped, en el caso del SARS-CoV-2, sus espigas actuarían como una llave para abrir la cerradura de entrada a las células que en su caso es la ECA-2.

Un estudio realizado por Zhou et al. indicó que la ECA-2 es probablemente el receptor celular de 2019-nCoV, que también fue el receptor de SARS-CoV y HCoV-NL6379.

\section{Hipertensión arterial, IECA, BRAT y COVID-1}

En un reporte reciente del brote de COVID-19 iniciado en diciembre de 2019 en la provincia china ${ }^{10}$ de Wuhan, Hubei, se muestra que las personas de edad avanzada y los portadores de enfermedad cardiovascular e hipertensión arterial desarrollan enfermedad más severa. La hipertensión arterial en pacientes que sufren de COVID-19 se ha reportado entre el 15 y 30\%, (en México en la actual etapa 2 del contagio, es mayor al $45 \%$ ) y en las series italianas hasta dos terceras partes de los que mueren, sufrian de hipertensión arterial. De acuerdo a esto podriamos afirmar los sujetos hipertensos, efectivamente parecen tener una enfermedad más grave, sin embargo en la mayoría de estos estudios ni la frecuencia ni la gravedad de la enfermedad están corregida por la edad y sabemos que la edad del paciente es el parámetro más sospechoso relacionado con la gravedad de la enfermedad viral, además llama la atención que esta relación no se ha descrito previamente para otro tipo de infecciones en pacientes que sufren hipertensión arterial ${ }^{11}$.

Los BRAT son los medicamentos antihipertensores más empleados en el mundo (en México los IECA los superan), ambos grupos de medicamentos aumentan la expresión de los receptores ECA-2 aproximadamente duplicándolos y hasta triplicándolos ${ }^{12}$, hasta ahora este hallazgo se ha realizado solo en animales y a pesar de que no existen estudios en humanos, en la mayoría de los estudios revisados, se extrapolan a los humanos los hallazgos en animales.

Curiosamente el único estudio que analiza el efecto del tratamiento con BRATs o IECAs en pacientes con COVID-19, marca exactamente lo contrario: se trata de un ensayo realizado en China, este es una revisión retrospectiva de los expedientes electrónicos médicos, de pacientes hospitalizados con COVID-19 del 11 de enero al 23 de febrero de 2020 en el Hospital Shenzhen Third People's. Se analizaron los resultados en 42 pacientes que se dividieron en dos grupos, 17 de ellos recibieron 
IECA o BRAT y otros 25 tratamiento antihipertensor que no los contenía. Los autores concluyen: "los pacientes que reciben terapia con IECA o BRAT tenían una tasa más baja de enfermedades graves y una tendencia hacia un nivel más bajo de IL- 6 en la sangre periférica. Además, la terapia IECA o BRAT aumentó el recuento de células T CD3 y CD8 en sangre periférica y disminuyó la carga viral máxima en comparación con otros medicamentos antihipertensivos. Esta evidencia respalda el beneficio del uso de IECA o BRAT para contribuir potencialmente a la mejora de los resultados clínicos de pacientes con hipertensión arterial COVID-19"13.

La posibilidad de que los BRAT y los IECA no sean dañinos, sino por lo contrario benéficos en casos de ataque agudo y severo viral al pulmón, se ha explorado desde antes de esta pandemia en pacientes con neumonía por COVID $-19^{14}$ y en casos SARS ${ }^{15}$

Pueden existir hipótesis alternas a la idea simple de que el aumento de receptores ECA-2 que produce el bloqueo del SRAA, resulte favorable a la infección y gravedad de la COVID-19 y por lo contrario este aumento de receptores sea favorable: como se indica en una publicación reciente ${ }^{16}$ la proteína del virus contenida en sus espigas induce una regulación a la baja de ECA-2 en el pulmón, lo cual origina una sobreproducción de At-II por la ECA, este exceso no es contrarrestado por la conversión de At-II a AT(1-7) por lo que el exceso de At-II provoca una severa vasoconstricción, aumento de la permeabilidad vascular, inflamación severa y fibrosis intersticial que explicaría la gravedad del daño que se produce en el pulmón.

Otra explicación alternativa similar a la anterior, que favorecería la hipótesis de que en realidad el empleo de agentes que actúan sobre el SRAA y que aumentan los receptores para ECA-2, sería el que el virus rápidamente ocupa todos los receptores ECA-2 disponibles (con mayor razón si han disminuido), dejando sin posibilidad a la Ang1-7 de efectuar su benéfica acción antinflamatoria y antifibrótica en los neumocitos, que puede ser vital para la preservación de la integridad anatómica y funcional de los alveolos pulmonares.

\section{Posición de distintas sociedades internacionales y mexicanas sobre el uso de IECA Y BRAT en pacientes con COVID-19.}

Después de la explosión mediática sobre los posibles peligros del tratamiento con antihipertensivos que actúan sobre el SRAASRA en pacientes de alto riesgo para adquirir la COVID-19 con aquellos con la enfermedad establecida, un importante grupo de sociedades científicas con interés en el campo, emitieron su opinión. Es de mencionarse que ninguna de ellas se inclina por suspender el tratamiento con IECA o BRAT por motivo de la COVID19.

La primera publicación al respecto es la de la Sociedad Europea de Hipertensión del 12 de marzo de 202017, seguida por la Sociedad Europea de Cardiología (Hypertension Council) ${ }^{18}$, Hypertension Canadá ${ }^{19}$, la Canadian Cardiovascular Society ${ }^{20}$, La Sociedad Renal del Reino Unido ${ }^{21}$, La Sociedad Intenacional de Hipertensión ${ }^{22}$, El Colegio Americano de Médicos (American College of Physicians) ${ }^{23}$, La Sociedad Española de Hipertensión ${ }^{24}$, En forma conjunta la Asociación Americana del Corazón (AHA), La Sociedad de Insuficiencia Cardiaca de América (HFSA) y el Colegio Americano de Cardiología $(\mathrm{ACC})^{25}$, Las Sociedades Europeas Renal y de Diálisis y Trasplante ${ }^{26}$, La Sociedad Americana de Nefrología Pediátrica. El Consejo Australiano de Presión Elevada ${ }^{27}$, La World Hypertension League ${ }^{28}$ La Sociedad Australiana de Diabetes ${ }^{29}$.En México el Grupo de Expertos en Tratamiento de la Hipertensión Arterial (GRETHA-México ${ }^{30}$, Sociedad Mexicana de Cardiología y Asociación Nacional de Cardiólogos de México ${ }^{31}$.

En todas estas declaraciones, se recomienda no retirar el tratamiento con IECA o BRAT en la población general por motivo de la pandemia, ni en pacientes que estén tomando estos medicamentos por cualquier indicación y que padezcan COVID-19, tampoco se indican como terapéutica para la COVID-19

\section{Conclusiones}

Por el momento y a falta de evidencia proveniente de estudios comparativos, los expertos y las sociedades médicas coinciden y recomiendan que, todo paciente que esté tomando medicamentos que actúan sobre el SRAA ya sea por hipertensión arterial, síndromes isquémicos coronarios y en especial insuficiencia cardiaca, no deben suspenderlos por motivo de la COVID-19, pero también se recomienda que en pacientes que no los están tomando, no se prescriban por razón de la COVID-19. El razonamiento ético se basa en que los beneficios del empleo de estos medicamentos en sus indicaciones aceptadas, está bien demostrado con evidencia sólida, mientras que el perjuicio o beneficio de su uso en pacientes con COVID-19 son actualmente hipótesis, sin evidencia razonable que las apoyen.

Una de las lecciones que nos reitera esta relación entre el COVID-19 y el SRAA, es el hecho que la biología no sigue las leyes humanas de la lógica general 
o del "sentido común", como sería el pensar de manera "muy lógica, que si el virus requiere para entrar a la célula del receptor ECA-2, y se aumenta la expresión por los medicamentos, aumenta las posibilidades del virus para infectar y dañar" y que la tentación de interpretar a la salud/enfermedad con estos instrumentos "lógicos" o "intuitivos", tan empleados por varios siglos por la Medicina y actualmente por no pocos legos.

Con una enfermedad de impacto catastrófico, como COVID-19 para la que no se han desarrollado vacunas, ni tratamiento específico, la desesperación y el buen deseo, predispone para aceptar hipótesis atractivas como las descritas y esto resulta en un error que puede ser muy peligroso y expresa la práctica de un pensamiento basado en ocurrencias y quizá buenos deseos, pero que no tiene cabida, en un pensamiento educado en la ciencia y que basa en especial sus decisiones profesionales en las evidencias mejores disponibles.

\section{Responsabilidades éticas}

Protección de personas y animales. Los autores declaran que para esta investigación no se han realizado experimentos en seres humanos ni en animales.

Confidencialidad de los datos. Los autores declaran que han seguido los protocolos de su centro de trabajo sobre la publicación de datos de pacientes.

Derecho a la privacidad y consentimiento informado. Los autores declaran que en este artículo no aparecen datos de pacientes.

\section{Bibliografía}

1. Wan Y, Shang J, Graham R, Baric RS, Li F. Receptor recognition by novel coronavirus from Wuhan: An analysis based on decade long structural studies of SARS. J Virology 2020; published online Jan 29. DOI:10.1128/ JVI.00127-20.

2. Sparks MA, Hiremath $S$ et al. "The Coronavirus Conundrum: ACE2 and Hypertension Edition"NephJC.http://www.nephjc.com/news/covidace2. Revisada 14 abril 2020

3. EslerM, Esler D. Can angiotensin receptor-blocking drugs perhaps be harmful in the COVID-19 pandemic'. J Hypertens. 2020 May;38(5)::781782. doi: $10.1097 / \mathrm{HJH} .0000000000002450$

4. https://www.dailymail.co.uk/news/article-8108735/Medicines-high-blood-pressure-diabetes-worsen-coronavirus-symptoms.html. Revisada 14 abril 2020

5. Karram T, Abbasi A, Keidar S, Golomb E, Hochberg I, Winaver J, et al. Effects of spironolactone and eprosartan on cardiac remodeling and angiotensin-converting enzyme isoforms in rats with experimental heart failure. Am J Physiol Heart Circ Physiol 2005;289:H1351-H1358.
6. Chappell MC, Marshall AC, Alzayadneh EM, Shaltout HA, Diz DI. Update on the ACE2-Angiotensin-(1-7)-MAS receptor axis. Front Endocrinol.2014; 4:201-215.

7. Zhu N, Zhang D, Wang W, Li X, Yang B, Song J, Zhao X, et al.A Novel Coronavirus from Patients with Pneumonia in China, 2019. N Engl J Med 2020;382:727-33.

8. Wrapp D, Wang N, Corbett KS, Goldsmith JA, Hsieh CL, Abiona O, Graham BS, McLellan JS. Cryo-EM structure of the 2019-nCoV spike in the prefusion conformation. Science. 2020 13;367:1260-3

9. Whu, N.; Zhang, D.; Wang, W.; Li, X.; Yang, B.; Song, J.; et al.. A Novel Coronavirus from Patients with Pneumonia in China, 2019. N. Engl. J. Med. 2020, 382, 727-33.

10. Wan Y, Shang J, Graham R, Baric RS, Li F. Receptor recognition by novel coronavirus from Wuhan: An analysis based on decade long structural studies of SARS. J Virology 2020; published online Jan 29. DOI:10.1128/ JVI.00127-20

11. Gutierrez F, Masia M, Mirete C, Soldan B, Rodriguez JC, Padilla S, et al. The influence of age and gender on the population based incidence of community-acquired pneumonia caused by different microbial pathogens. $\mathrm{J}$ Infect 2006;53:166-74.

12. Ferrario $C M$, Jessup J, Chappell MC, Averill DB, Brosnihan KB Tallant EA, et al. Effect of angiotensin-converting enzyme inhibition and angiotensin II receptor blockers on cardiac angiotensin-converting enzyme 2. Circulation 2005; 111:2605-10.

13. Meng J, Xiao G, Zhang J, He X, Ou M, Bi J, et al. Renin-angiotensin system inhibitors improve the clinical outcomes of COVID-19 patients with hypertension. Emerging Microbes \& Infections. 2020; 9:1, 757-60,

14. Sun ML, Yang JM, Sun YP, Su GH. [Inhibitors of RAS might be a good choice for the therapy of COVID-19 pneumonia]. Zhonghua Jie $\mathrm{He} \mathrm{He}$ $\mathrm{Hu}$ Xi Za Zhi. 2020;43:219-22.

15. Kuba K, Imai Y, Rao S, Gao H, Guo F, Guan B, et al. A crucial role of angiotensin converting enzyme 2 (ACE2) in SARS coronavirus-induced lung injury. Nat Med 2005;11:875-9

16. Battistoni A, Volpe M. Might renin-angiotensin system blockers play a role in the COVID-19 pandemic?. Eur Heart J - Cardiovascular Pharmacotherapy. Published on line 04-12-20 doi:10.1093/ehjcvp/pvaa030

17. https://www.eshonline.org/spotlights/esh-stabtement-on-covid-19-2/. Consultada 14 abril 2020

18. https://www.escardio.org/Councils/Council-on-Hypertension-(CHT)/ News/position-statement-of-the-esc-council-onhypertension-on-ace-inhibitors-and-ang. Consultada 14 abril 2020.

19. https://joel-topf-1vjl.squarespace.com/config/. Consultada 14 abril 2020.

20. https://www.ccs.ca/images/Images_2020/CCS_CHFS_Update_COVID_ CV_medications_Mar20.pdf. Consultada 14 abril 2020.

21. https://renal.org/covid-19/checklist-renal-services-respect-covid-19-pandemic/), International Society of Hypertension (https://ish-world.com/ news/a/A-statement-from-the-International-Society-of-Hypertension-on-COVID-19/), Consultada 14 abril 2020.

22. https://ish-world.com/news/a/A-statement-from-the-International-Society-of-Hypertension-on-COVID-19/), Consultada 14 abril 2020.

23. https://assets.acponline.org/coronavirus/scormcontent/? $\mathrm{ga}=2.70091792 .1486472653 .1584392833-1102360168.1584392833 \# /$ Consultada 14 abril 2020.

24. https://www.seh-lelha.org/covid-19/.Consultada 14 abril 2020.

25. https://newsroom.heart.org/news/patients-taking-ace-i-and-arbs-whocontract-covid-19-should-continue-treatment-unless-otherwise-advised-by-theirphysician\#.XnDMEt7Ot9l.twitter Consultada 14 abril 2020.

26. https://www.era-edta.org/en/covid-19-news-and-information/\#toggl e-id-1 Consultada 14 abril 2020.

27. https:/www hbprca com.au/wp-content/uploads/2020/03/HBPRCA-Statement-on-COVID-19-and-BP-medication-17.03.20.pdf Consultada 14 abril 2020.

28. https://www.whleague.org/index.php/2014-07-09-22-47-11/covid-19 hypertensionguidance. (30 March 2020). Consultada 14 abril 2020.

29. https://diabetessociety.com.au/downloads/20200329\%20ADS\%20Letter\%20re\%20COVID-19\%20and\%20Diabetes\%20HPs\%2029032020_ Update\%20.pdf Consultada 14 abril 2020.

30. https://ampac.org.mx/wp-content/uploads/2020/04/COMUNICADO-GRETHA-SOBRE-LA-RELACIOi\%CC\%80N-ENTRE-COVID.pdf.pdf.pdf.pdf Consultada 14 abril 2020.

31. https://ancam.org.mx/\#/comunicados/2-covid-19 Consultada 14 abril 2020. 